\title{
Perfil de marcadores do estresse oxidativo em caprinos suplementados ou não com selênio e vitamina $\mathrm{E}$ e submetidos à insulação escrotal
}

\section{Oxidative stress profile in goats supplemented or not with selenium and vitamin $\mathbf{E}$ and submitted to scrotal insulation}

\author{
Janaína Azevedo Guimarães ${ }^{1}$; Guadalupe Carvalho Xavier ${ }^{2}$; Pierre Castro \\ Soares $^{3 *}$; Alexandre Cruz Dantas ${ }^{1}$; Filipe Aureliano Pedrosa Soares ${ }^{4}$; Carla Lopes \\ Mendonça ${ }^{1}$; Maria Madalena Pessoa Guerra ${ }^{3}$; Valdemiro Amaro Silva Júnior ${ }^{5}$
}

\begin{abstract}
Resumo
Para avaliar o efeito da suplementação com selênio e vitamina E na dieta de caprinos induzidos à insulação escrotal (IE) sobre o perfil de indicadores bioquímicos do metabolismo oxidativo sistêmico foram utilizados 12 animais, os quais foram distribuídos aleatoriamente em dois grupos: G1 - sem suplementação; G2 suplementados com selênio $(0,1 \mathrm{mg} / \mathrm{Kg}$ de peso vivo de selenito de sódio) e vitamina $\mathrm{E}(0,3 \mathrm{UI} / \mathrm{Kg}$ de peso vivo). Ao término do período de adaptação de 30 dias, realizou-se a indução da insulação escrotal (IE) com colocação de bolsas plásticas nos testículos por período de 18 dias. A suplementação com Se e Vitamina E teve início 60 dias antes da indução da IE e mantida durante 42 dias após o término da IE, correspondendo à fase pós-insulação (PIE). Amostras de sangue foram obtidas por venopunção jugular para obtenção de sangue total para análise de glutationa reduzida (GSH) e plasma para análise de substância reativa ao ácido tiobarbitúrico (TBARS) e determinação da habilidade de redução férrica plasmática (HRFP). Não houve efeito da suplementação dietética com Se e Vitamina E sobre o perfil do conjunto de variáveis estudadas, porém verificou-se ação oxidativa sistêmica compreendida pela diminuição significativa da GSH no período da IE, com aumento gradativo no período PIE, bem como menores valores médios da variável HRFP observados no período da IE e maiores médias no período de PIE. Concluiu-se que análise da atividade da glutationa reduzida (GSH) e das concentrações de substâncias reativas ao ácido tiobarbitúrico (TBARS) e habilidade de redução férrica plasmática (HRFP) são métodos laboratoriais recomendados para avaliação da resposta aos danos oxidativos sistêmicos causados pelo estresse térmico testicular em caprinos.
\end{abstract}

Palavras-chave: Testículo, caprino, estresse térmico, enzimas, antioxidantes

\begin{abstract}
To evaluate the effect of supplementation with selenium and vitamin $\mathrm{E}$ in the diet of goats induced to scrotal insulation (SI) on the profile of biochemical markers of systemic oxidative metabolism 12 animals were randomly divided into two groups: G1 - no supplementation G2 - supplemented with selenium $(0.1 \mathrm{mg} / \mathrm{kg}$ body weight of sodium selenite) and vitamin E ( $0.3 \mathrm{IU} / \mathrm{kg}$ body weight). At the end of the adjustment period of 30 days, the scrotal insulation (SI) was performed with the placement
\end{abstract}

\footnotetext{
1 Médicos Veterinários, Clínica de Bovinos, Campus Garanhuns, Universidade Federal Rural de Pernambuco, UFRPE. E-mail: nainalago@yahoo.com.br; vetacdantas@gmail.com; carlalopes.mendonça@gmail.com

2 Médica Veterinária Autônoma. E-mail: mpguerra@dmv.ufrpe.br

3 Profs. do Dept ${ }^{\circ}$ de Medicina Veterinária, UFRPE. E-mail: psoares@dmv.ufrpe.br; mpguerra@dmv.ufrpe.br

4 Discente em Medicina Veterinária, Dept ${ }^{0}$ de Medicina Veterinária. E-mail: filipinho_soares@hotmail.com

5 Prof. do Dept ${ }^{\text {}}$ de Morfologia e Fisiologia Animal,UFRPE. E-mail: valdemiroamaro@gmail.com

* Autor para correspondência
} 
of plastic bags in the testes for 18 days. Supplementation with Se + vitamin E began 60 days before induction of SI and maintained for 42 days after the end of SI, corresponding to post-insulation (PSI). Blood samples were obtained by jugular venipuncture to obtain total blood for analysis of reduced glutathione (GSH) and plasma for analysis of thiobarbituric acid reactive substance (TBARS) and determination of ferric reducing ability of plasma (FRAP). No effect of dietary supplementation with $\mathrm{Se}+$ vitamin $\mathrm{E}$ on the profile of all the variables studied, but there was systemic oxidative action understood by a significant decrease of GSH during the SI, with a gradual increase in the PSI period, as well as lower average of FRAP observed during the SI and the highest average in the period from PSI. It was concluded that analysis of the activity of reduced glutathione (GSH) and concentrations of thiobarbituric acid reactive substances (TBARS) and ferric reducing ability of plasma (FRAP) are recommended laboratory methods to evaluate the systemic response to oxidative damage caused by thermal stress testis in goats.

Key words: Testis, goat, heat stress, enzymes, antioxidants

\section{Introdução}

Como conhecido, as variações estacionais provocam mudanças na atividade sexual dos machos e na produção qualitativa e quantitativa do sêmen por meio da interação de diferentes fatores como: disponibilidade de alimentos, temperatura ambiental, fotoperíodo e atividade hipofisária (JENNINGS, 1976). Dentre esses, destaca-se a temperatura ambiental, uma vez que a intensidade do calor pode provocar falhas nos mecanismos de termorregulação testicular, favorecendo a degeneração do epitélio seminífero e, conseqüentemente, a espermatogênese (SANTOS; SIMPLÍCIO; MACHADO, 1998). Pesquisas recentes têm sugerido o envolvimento do estresse oxidativo como indutor dos danos provocados pelo estresse térmico testicular (NICHI et al., 2006; XAVIER et al., 2008; PAUL; TENG; SAUNDERS, 2009).

Em condições experimentais, a insulação escrotal promove alterações degenerativas testiculares (FLORENTINO et al., 2003a), uma vez que o aumento da temperatura do escroto, quer seja em condição natural ou induzido pela insulação, interfere na termorregulação testicular, predispondo à degeneração do epitélio germinativo gonadal (SANTOS; SIMPLÍCIO, 2000). Os mecanismos através dos quais ocorre morte celular em resposta ao estresse calórico ainda não estão completamente elucidados, no entanto alguns estudos têm demonstrado que ocorre morte programada das células germinativas através de apoptose. Este tipo de morte celular também ocorre em função da ação de substâncias oriundas do estresse oxidativo, embora não seja bem conhecida a origem das espécies reativas de oxigênio (EROs) nos casos de estresse calórico, mas se sabe que estão aumentadas nestes casos (KUMAGAI et al., 2002).

As lesões estruturais testiculares são marcantes quando altas temperaturas incidem sobre este órgão e seguramente as conseqüências não se restringem apenas ao testículo, mas também ao organismo como um todo, uma vez que é acompanhado do aumento na produção de radicais livres, determinando alterações no metabolismo celular sistêmico, pois, de acordo com Sies (1993), espécies oxidativas podem ser formadas no interior das células e serem transportadas para outroslocais onde poderão ser ativadas e provocar oxidação de componentes celulares.

Em relação à lipoperoxidação causada pelas EROs, verifica-se que esta tem sido o processo mais extensivamente estudado (FONTEQUE, 2005). No organismo as EROs reagem com ácidos graxos poliinsaturados para formar lipoperóxidos que compõem a cascata de reações degenerativas dos lipídeos (BIESALSKY, 2002). Como conseqüência deste efeito, os 
radicais peroxil lipídicos são liberados para a circulação sangüínea elevando sua concentração, indicando a ocorrência de lesão de membrana celular (YAGI, 1987). Daí a importância de avaliar o metabolismo oxidativo por meio de provas laboratoriais utiizando-se indicadores bioquímicos capazes de demonstrar a condição orgânica de animais sob diferentes situações clínicas, como por exemplo, animais sob condição de estresse térmico testicular.

Danos tissulares, induzidos por estresse oxidativo, são minimizados por elementos de defesa, ou seja, por antioxidantes. Dentre os mecanismos de defesa, estão reconhecidamente envolvidos na proteção das membranas biológicas contra a lipoperoxidação o selênio e a vitamina E, os quais atuam sinergicamente (UNDERWOOD; SUTTLE, 1999). A vitamina $E$ previne a peroxidação lipídica das membranas biológicas pelas EROs nos estágios iniciais, enquanto que o selênio atua como componente da enzima glutationa peroxidase a qual reduz o peróxido de hidrogênio e radicais hidroxil a produtos menos reativos (VAN METRE; CALLAN, 2001). O uso de nutrientes antioxidantes, como o selênio e a vitamina $\mathrm{E}$, tem alcançado interesse por parte da comunidade científica em escala nos inúmeros modelos experimentais e ensaios clínicos registrados, verificando-se os efeitos benéficos que estes nutrientes estabelecem para os animais (UNDERWOOD; SUTTLE, 1999).

Não se tem conhecimento de nenhuma pesquisa realizada no campo da bioquímica clínica de caprinos sob estresse térmico testicular, a fim de determinar, com acurácia, os efeitos desse fator de estresse sobre o organismo como um todo, principalmente, no que se refere à produção de espécies reativas de oxigênio. Santos e Simplício (2000), Florentino et al. (2003b), Xavier et al. (2008), demonstraram que a ocorrência de processo degenerativo é marcante em caprinos quando induzidos a insulação escrotal, porém o conjunto de variáveis avaliadas foi particular ao parênquima testicular e ao sêmen.

Sendo assim, objetivou-se avaliar o perfil sanguíneo de indicadores bioquímicos de estresse oxidativo sistêmico (glutationa reduzida - GSH, Substâncias Reativas ao Ácido TiobarbitúricoTBARS e Habilidade de Redução Férrica Plasmática- HRFP) antes, durante e após a fase de insulação escrotal em caprinos suplementados com selênio e vitamina E.

\section{Material e Métodos}

Foram selecionados, após exame andrológico, 12 machos da espécie caprina, mestiços, com idade variando entre sete e oito meses, os quais foram sorteados, por amostragem probabilística, para composição de dois grupos: G1 (não suplementados; $\mathrm{n}=6$ ) e G2 (suplementados com selênio e vitamina $E ; n=6)$.

Os animais foram mantidos em regime de confinamento em baias individuais. A dieta dos animais foi constituída de uma mistura concentrada à base de milho triturado, farelo de soja (Glycine híspida, Maxin) e sal comum $(\mathrm{NaCl})$ acrescido de calcário, além de água $a d$ libitum. A ração foi fornecida duas vezes ao dia, sendo $200 \mathrm{~g} /$ cabeça/dia pela manhã e $200 \mathrm{~g} /$ cabeça/dia à tarde. Com relação ao feno triturado de capim Tifton (Cynodon dactylon), este foi oferecido à vontade, porém permitindo sobras de $10 \%$, com base em pesagens consecutivas das sobras do dia anterior.

O período de adaptação das instalações e da dieta base foi de 30 dias. Ao término deste período, foi realizada a suplementação por via oral de Selênio e Vitamina E aos animais do G2, cujo período da oferta foi de 60 dias. O selênio foi suplementado sob forma de selenito de sódio, na dose de $0,1 \mathrm{mg} / \mathrm{Kg}$ de peso vivo, e a vitamina $\mathrm{E}$ na dose de $0,3 \mathrm{UI} / \mathrm{Kg}$ de peso vivo. Os animais foram pesados em intervalos de seis dias para correção da suplementação, com base 
na regressão linear do ganho de peso em função do tempo. Os animais do G1 apenas receberam a dieta base e o placebo foi constituído de farelo de soja e milho triturado.

Decorridos 90 dias correspondentes de adaptação e suplementação, os animais de ambos os grupos foram induzidos à insulação escrotal (IE). A IE foi realizada com o emprego de bolsa plástica de polietileno de dupla parede envolvendo os testículos, separadas por uma camada de algodão de aproximadamente cinco $\mathrm{mm}$, conforme Florentino et al. (2003a). Esta fase teve duração de 18 dias. Ao término do período de IE, quando foram retiradas as bolsas plásticas dos testículos, foram escolhidos novamente, por amostragem probabilística, três animais de cada grupo. Os demais animais ( $\mathrm{G} 1=3$ e $\mathrm{G} 2=3$ ) continuaram nas mesmas condições de recebimento da dieta base (G1 e G2) e da suplementação (G2) por período adicional de 42 dias, correspondendo à fase Pós-Insulação Escrotal (PIE). O período total de experimento foi de 150 dias.

Amostras de sangue foram coletadas por venopunção jugular, em tubos siliconizados com sistema a vácuo, sem anticoagulante e com anticoagulante (ácido etilenodiaminotetracético - EDTA), para obtenção do soro, plasma e sangue total, respectivamente. Os tubos de sangue sem anticoagulante foram mantidos à temperatura ambiente para retração do coágulo e obtenção de soro, enquanto que os demais tubos com anticoagulante foram refrigerados e encaminhados ao laboratório para posterior processamento analítico. As coletas foram efetuadas ao término de 90 dias do período de adaptação e suplementação (0d); no período de IE (6, 12 e 18 dias); no período PIE (7, 14, 21, 28,35 e 42 dias), totalizando 10 coletas.

A atividade da GSH foi determinada por método colorimétrico descrito por Beutler, Duron e Kelly (1963), utilizando-se sangue total. As leituras foram efetuadas em espectrofotômetro em absorbância de $412 \mathrm{~nm}$. Como esta técnica fornece resultado em densidade óptica o mesmo foi transformado em $\mathrm{mg} / 100 \mathrm{~mL}$ de sangue, realizando-se o seguinte cálculo: GSH (mg/ 100 $\mathrm{mL}$ de sangue $)=(\mathrm{OD} 1-\mathrm{OD} 2) \times 31040 / \mathrm{VG}$. Onde, OD1 = Densidade ótica inicial, OD2 = Densidade ótica final e $\mathrm{VG}=$ Volume globular da amostra. A concentração de TBARS foi determinada no soro pelo método descrito por Esterbauer e Cheeseman (1990). A leitura foi realizada em espectrofotômetro em absorbância de 532nm. A Habilidade de Redução Férrica Plasmática foi realizada empregando-se plasma, de acordo com o método colorimétrico descrito por Benzie e Strain (1996), que relataram a HRFP, em pH ácido, reduzindo o complexo tripiridiltiazina férrica $(\mathrm{Fe} 3+-\mathrm{TPTZ})$ à forma ferrosa $\left(\mathrm{Fe}^{2+}\right)$, formando uma coloração azul intensa. Esta foi determinada por espectrofotômetro em absorbância de 593nm.

Os parâmetros foram testados, inicialmente, quanto à sua distribuição normal, e foi necessário submeter à variável TBARS à transformação pela raiz quadrada $[\mathrm{RQ}(\mathrm{x}+1)]$. Os dados que atenderam as premissas de normalidade ou transformados foram submetidos à análise de variância (Teste F) que separou como causa de variação efeitos de grupos, dias de coleta e interação grupos $\mathrm{x}$ coletas. Foi aplicado o nível de significância (P) de 5\%, e a diferença mínima significativa (DMS) do teste de Tukey para comparações de médias quando na presença de significância. Os dados foram analisados por meio do programa computacional Statistical Analysis System (SAS, 2010). O referido projeto foi aprovado pelo Comitê de Ética do Departamento de Medicina Veterinária (Processo: 6260/2006 e Resolução: 88/2006, em 31.05.2006) e pelo Conselho de Ensino, Pesquisa e Extensão (Resolução 302/2006, em 18.09.2006). 


\section{Resultados e Discussão}

Não se observou variação significativa da atividade da GSH entre os grupos (P > 0,0969), para os grupos controle e suplementados com selênio e vitamina $\mathrm{E}$, respectivamente. Não houve interação grupos $\mathrm{x}$ tempos de coleta $(\mathrm{P}>0,3740)$. Quanto aos tempos de coleta, estes sofreram variação significativa $(\mathrm{P}<$ de 0,0002$)$. Maiores valores médios da atividade da GSH eritrocitária foram identificados no momento anterior a IE e no período PIE. Menores valores foram registrados no período da IE, compreendido entre o $6^{\circ}$ e $18^{\circ}$ dias da IE (Tabela 1).

Durante o período de indução da insulação escrotal, ocorreu diminuição na atividade da GSH sendo explicada pela ação dos radicais livres que penetram na hemácia e se ligam aos radicais sulfidrilas, presentes na membrana citoplasmática, formando grupos dissulfetos que danificam a parede celular (HOWELL; GOONERATNE, 1987). Os valores encontrados no momento inicial da coleta de sangue, para ambos os grupos, estiveram pouco abaix o do limite considerado normal, pois de acordo com Vallejo, Tunón e Gonzáles (1989), o limite mínimo desta enzima em caprinos é $60 \mathrm{mg} / 100 \mathrm{~mL}$ de sangue. Moreno et al. (1987), observaram que existe um fator genético que determina a concentração de GSH em caprinos, assim como observado em ovinos por Igbokwe e Bah (1999), onde existe um polimorfismo da GSH que classifica os animais em dois tipos, os que apresentam concentração de GSH abaixo de $60 \mathrm{mg} / 100 \mathrm{~mL}$ de sangue e os que apresentam concentrações acima de $60 \mathrm{mg}$ de GSH/100mL de sangue.

Verificou-se que no período PIE os valores da GSH elevaram-se progressivamente e ao término do período experimental os dois grupos recuperaram as concentrações iniciais de GSH. Este fato é explicado pela ação da Glutationa Redutase, a qual que tem a função de regenerar a GSH reduzida, com o objetivo de impedir a inativação do ciclo metabólico da glutationa no interior dos eritrócitos (ROVER JÚNIOR; HÖEHR; VELLASCO, 2001). Considerandose a maior atividade de GSH no período PIE, entende-se que houve uma efetiva reperfusão tecidual acompanhada da diminuição da ação dos peróxidos e menor consumo de GSH. Melhores respostas poderiam ter sido observadas caso fosse aumentada a dosagem do selênio, uma vez que, de acordo com Underwood e Suttle (1999), as necessidades diárias de selênio para um pequeno ruminante variam de 0,1 a $0,3 \mathrm{mg} / \mathrm{kg}$ e neste experimento foi administrada a menor dose. Outro fator importante é a forma química do elemento a ser administrado, onde a utilização do selênio como selenoaminoácido (selenometionina ou selenocisteína) melhora a retenção do mesmo no organismo (FOSTER; SUMAR, 1997), e que neste delineamento experimental foi utilizado o selenito de sódio.

Quanto aos TBARS, não foi verificado variação significativa para os fatores grupos $(\mathrm{P}>0,3275)$, tempos de coleta $(\mathrm{P}>0,4685)$ e interações grupos $\mathrm{x}$ tempos de coleta $(\mathrm{P}>0,3275)$ nos caprinos induzidos a estresse térmico testicular e suplementados ou não com selênio e vitamina E. Embora não se tenha observado diferenças entre grupos, a concentração de TBARS no grupo sem suplementação apresentou médias mais elevadas, indicando que não houve grau de peroxidação lipídica capaz de determinar elevados valores deste indicador no sangue. A presença de médias mais elevadas no final do período de IE confirma a presença de peroxidação lipídicas. Segundo Strauss (1981) e Antunes et al. (2008), esta substância é o produto final da oxidação pelos radicais livres dos lipídeos. É de supor que doses maiores tanto do selênio quanto da vitamina E pudessem reduzir a reação em cascata da formação de radicais livres. 


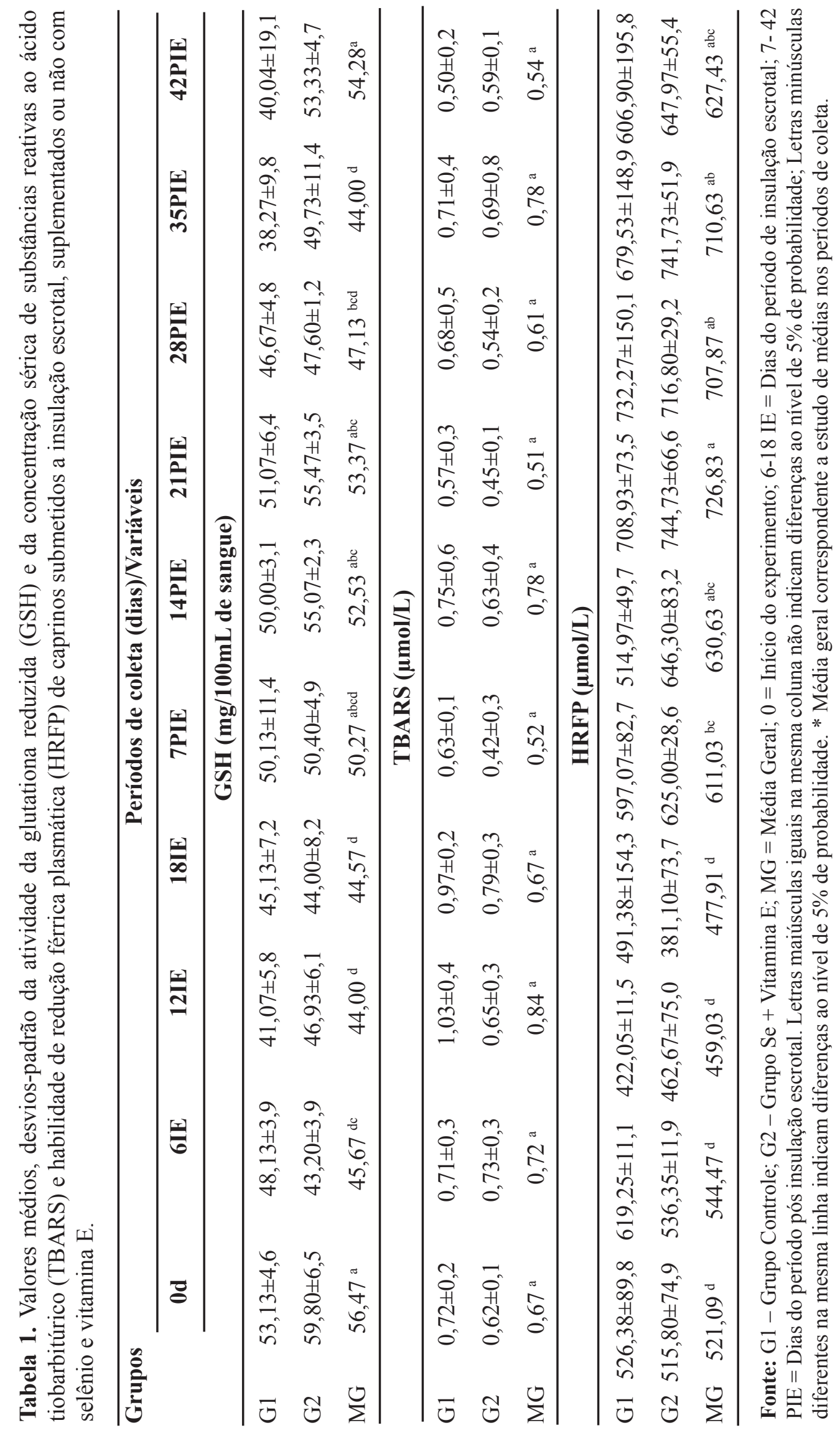


De acordo com Beconi et al. (1991), os níveis de TBARS podem ser influenciados pela quantidade de células susceptíveis a peroxidação lipídica, e neste caso o estresse calórico induzido ao nível dos testículo refletiu no perfil de estresse oxidativo sistêmico. Não existem valores de referência deste metabólito para caprinos, porém, em trabalho desenvolvido com cabras, para avaliar o efeito da suplementação de vitamina E no desempenho reprodutivo de fêmeas submetidas a protocolo de sincronização estral com uso de esponjas intravaginais, foi encontrado valor basal médio semelhante ao encontrado neste estudo (SÖNMEZ et al., 2009).

Não foi registrado efeito de grupos em relação à HRFP ( $\mathrm{P}>0$ 0,3813), para os grupos controle e suplementados com selênio e vitamina E, respectivamente. Diferenças de médias entre os tempos de coleta foram identificadas ( $\mathrm{P}<0,0001)$. Quanto à interação grupos x momentos, este não foi significativo $(\mathrm{P}>0,9865)$. Menores valores de médias da HRFP foram identificados no período da IE, identificando-se maiores médias no período PIE (Tabela 1). Estes resultados podem ser explicados pela depleção das reservas de antioxidantes do plasma no auge do estresse oxidativo, uma vez que coincide com os momentos em que ocorreu diminuição dos níveis da GSH. Guimarães et al. (2007), avaliaram a HRFP em ratos e não encontraram diferença significativa nos valores de HRFP destes animais submetidos a torção do cordão espermático, embora tenham observado redução da atividade de GSH nos momentos de maior estresse oxidativo.

Após o período de IE foi observado um aumento da capacidade antioxidante plasmática representada pela HRFP, indicando que o organismo foi estimulado a produzir antioxidantes mediante o desafio imposto, estando associado também ao término do insulto térmico testicular. Weigel, Ortolani e Sucupira (2010) também observaram este comportamento na concentração de HRFP de ovinos intoxicados por cobre, onde os maiores índices desta variável foram encontrados dois dias após a crise hemolítica. Ao término do período experimental os valores médios da HRFP ainda não haviam atingido os valores basais, confirmando a contínua produção de antioxidantes, embora os demais compostos analisados (GSH e TBARS) tivessem retornado aos valores basais.

Embora não tenha sido encontrado na literatura valores de referência da HRFP para a espécie caprina, pode-se crer que esta foi eficiente em avaliar a capacidade antioxidante plasmática. Weigel, Ortolani e Sucupira (2010), trabalhando com ovinos intoxicados experimentalmente com cobre encontrou valores basais semelhantes aos observados neste experimento. Outros estudos são sugeridos no sentido de averiguar o grau de relação existente entre esta variável com outras utilizadas como indicadores do metabolismo oxidativo, em situações de estresse térmico testicular e em outras enfermidades de interesse pecuário. Não foram encontradas referências para explicar o aumento sistêmico das EROs em experimentos com IE em caprinos. No entanto, pode-se supor que as EROs produzidas nos testículos foram liberadas e agiram sobre os eritrócitos, causando ativação de substâncias antioxidantes intra-eritrocitárias, como a GSH. Esta suposição baseia-se na afirmação de Sies (1993), onde uma ERO pode se formar em um determinado órgão e provocar lesão em outro.

Análise da atividade da glutationa reduzida (GSH) e das concentrações de substâncias reativas ao ácido tiobarbitúrico (TBARS) e habilidade de redução férrica plasmática (HRFP) são métodos laboratoriais recomendados para avaliação da resposta aos danos oxidativos sistêmicos causados pelo estresse térmico testicular em caprinos.

\section{Agradecimentos}

Á FACEPE pela aprovação do referido projeto por meio do Edital FACEPE/MCT/CNPq/CT-Infra e concessão de bolsa de mestrado. 


\section{Referências}

ANTUNES, M. V.; LAZZARETTI, C.; GAMARO, D. D.; LINDEN, R. Estudo pré-analítico e de validação para determinação de malondialdeído em plasma humano por cromatografia líquida de alta eficiência, após derivatização com 2,4-dinitrofenilhidrazina. Revista Brasileira de Ciências Farmacêuticas, São Paulo, v. 44, n. 2, p. 279-287, 2008.

BECONI, M. T.; AFFRANCHINO, M. A.; SCHANG, L. M.; BEORLEGUI, N. B. Influence of antioxidants on SOD activity in bovine sperm. Biochemistry International, Sydney, v. 23, n. 3, p. 545-553, 1991.

BENZIE, I. F. F.; STAIN, J. J. The ferric reducing ability of plasma (FRAP) as a measure of "Antioxidant Power": the FRAP assay. Analytical Biochemistry, Orlando, v. 239, n. 1, p. 70-76, 1996.

BEUTLER, E.; DURON, O.; KELLY, B. M. Improved method for the determination of blood glutathione. Journal of Laboratory and Clinical Medicine, St. Louis, v. 61, n. 5 , p. $882-888,1963$.

BIESALSKY, H. K. Free radicals theory of aging. Current Opinion in Clinical Nutrition Metabolic Care, Amsterdam, v. 5, n. 1, p. 5-10, 2002.

ESTERBAUER, H.; CHEESEMAN, K. H. Determination of aldehydic lipid peroxidation products: malonaldehyde and 4-hydroxynonenal. Methods in Enzymology, New York, v. 186, n. 1, p. 407-421, 1990.

FLORENTINO, C. M.; REIS, J. C.; GUERRA, M. M. P.; MAIA, F. C.; COLETO, Z. F. Efeito do tempo de insulação escrotal sobre a estrutura do parênquima testicular de caprinos (Capra hircus, L) sem raça definida. Ciência Veterinária nos Trópicos, Recife, v. 6, n. 1, p. 29-38, 2003a.

FLORENTINO, C. M.; REIS, J. C.; GUERRA, M. M. P.; SOUZA, A. F.; MERGULHÃO, F. C. C.; BATISTA, A. M.; COLETO, Z. F. Efeito do tempo de insulação escrotal sobre os constituintes do plasma seminal de caprinos (Capra hircus, L) sem raça definida. Ciência Veterinária nos Trópicos, Recife, v. 6, n. 1, p. 39-45, $2003 \mathrm{~b}$.

FONTEQUE, J. H. Estress oxidativo e lipoperoxidação devido a anemia induzida por perda aguda de sangue em ovinos. 2005. Tese (Doutorado em Medicina Veterinária) - Faculdade de Medicina Veterinária e Zootecnia da Universidade Estadual Paulista, Botucatu, São Paulo.

FOSTER, L. H.; SUMAR, S. Selenium in health and disease: a review. Critical Reviews in Food Science and Nutrition, London, v. 37, n. 3, p. 211-228, 1997.

GUIMARÃES, S. B.; ARAGÃO, A. A.; SANTOS, J. M. V.; KIMURA, O. S.; BARBOSA, P. H. U.;
VASCONCELOS, P. R. L. Oxidative stress induced by torsion of the spermatic Cord in Young rats. Acta Cirúrgica Brasileira, São Paulo, v. 22, n. 1, p. 30-33, 2007.

IGBOKWE, I. O.; BAH, G. S. Erythrocyte glutathione concentrations in Nigerian sheep in the Sahel region. Revue d'Elevage et de Medecine Veterinaire des Pays Tropicaux, Nigeria, v. 52, n. 1, p. 77-80, 1999.

JENNINGS, J. J. Effect of season and mating frequency on semen characteristics in rams. In: INTERNATIONAL CONGRESS OF ANIMAL REPRODUCTION AND ARTIFICIAL INSEMINATION, 1., 1976, Kracow. Anais... Kracow: The Polish Society for Animal Reproduction, 1976. p. 998-1001.

KUMAGAI, A.; KODAMA, H.; KUMAGAI, J.; FUKUDA, J.; KAWAMURA, K.; TANIKAWA, H.; SATO, N.; TANAKA, T. Xanthine oxidase inhibitors suppress testicular germ cell apoptosis induced by experimental cryptorchidism. Molecular Human Reproduction, Oxford, v. 8, n. 1, p. 118-123, 2002.

MORENO, A.; HABA, M.; MORERA, L; RODERO, A. Erithrocyte GSH concentration in the Malagueña goat breed. Archivos de Zootecnia, Córdoba, v. 36, n. 136, p. 313-319, 1987.

NICHI, M.; BOLS, P. E. J.; ZUGE, R. M.; BARNABE, V. H.; GOOVAERTS, I. G. F.; CORTADA, C. N. M . Seasonal variation in semen quality in Bos indicus and Bos taurus bulls raised under tropical conditions. Theriogenology, New York, v. 66, n. 4, p. 822-828, 2006.

PAUL, C.; TENG, S.; SAUNDERS, P. T. K. A single, mild, transient scrotal heat stress causes hypoxia and oxidative stress in mouse testes, which germ cell death. Biology of Reproduction, New York, v. 80, n. 5, p. 913919, 2009.

ROVER JÚNIOR, L.; HÖEHR, N. F.; VELLASCO, A. P. Sistema antioxidante envolvendo o ciclo metabólico da glutationa associado a métodos eletroanalíticos na avaliação do estresse oxidativo. Química Nova, São Paulo, v. 24, n. 1, p. 112-119, 2001.

SANTOS, D. O.; SIMPLÍCIO, A. A. Parâmetros escrototesticulares e de sêmen em caprinos adultos submetidos à insulação escrotal. Pesquisa Agropecuária Brasileira, Brasília, v. 35, n. 9, p. 1835-1841, 2000.

SANTOS, D. O.; SIMPLÍCIO, A. A.; MACHADO, R. Características escroto-testiculares e do ejaculado em bodes mestiços submetidos à insulação escrotal. Arquivo Brasileiro de Medicina Veterinária e Zootecnia, Belo Horizonte, v. 50, n. 3, p. 287-291, 1998.

SAS Institute. SAS/STAT User's guide. Version 8.2. 6. ed. Cary: Statistical Analysis System Institute, NC, USA, 
2010.

SIES, H. Strategies of antioxidant defense. European Journal Biochemistry, Berlin, v. 215, n. 2, p. 213-219, 1993.

SÖNMEZ, M.; BOZKURTA, T.; TÜRKA, G.; GÜRA, S.; KIZILB, M.; YÜCEB A. The effect of vitamin E treatment during preovulatory period on reproductive performance of goats following estrous synchronization using intravaginal sponges. Animal Reproduction Science, Amsterdam, v. 114, n. 1/3, p. 183-192, 2009.

STRAUSS, R. G. Malonaldehyde formation is not a suitable screening test to detect oxidation in human neutrophils. Journal Clinical of Pathology, London, v. 34, n. 7, p. 800-802, 1981.

UNDERWOOD, E. J.; SUTTLE, N. F. The mineral nutrition of livestock. 3. ed. Wallingford: Cabi Publishing, 1999. $614 \mathrm{p}$.

VALLEJO, M.; TUNÓN, M. J.; GONZÁLES, P. Interacciones entre parámetros sanguíneos (HB, $\mathrm{GSH}$, hematocrito, Ke e Kp) en caprinos. Archivos de Zootecnia, Córdoba, v. 38, n. 141, p. 177-188, 1989.

VAN METRE, D. C.; CALLAN, R. J. Selenium and vitamin E. Veterinary Clinics of North American: Food Animal Practice, Philadelphia, v. 17, n. 2, p. 373-402, 2001.

WEIGEL, R. A.; ORTOLANI, E. L.; SUCUPIRA, M. C. A. Avaliação do metabolismo oxidativo de ovinos intoxicados por cobre e tratados com tetratiomolibdato associado ou não a vitaminas antioxidantes. Brazilian Journal of Veterinary Research and Animal Science, São Paulo, v. 47, n. 6, p. 421-428, 2010.

XAVIER, G. C.; MAYMONE, A. C. M.; SOARES, P. C.; SILVA JÚNIOR, V. A.; GUERRA, M. M. P. Suplementação dietética com Selênio e Vitamina E nos parâmetros seminais de caprinos induzidos à insulação escrotal. Acta Scientiarum. Animal Sciences, Paraná, v. 30, n. 1, p. 103-111, 2008.

YAGI, K. Lipid peroxides and human diseases. Chemistry and Physics of Lipids, Amsterdam, v. 45, n. 2/4, p. 337351, 1987. 
